\title{
The Rook Monoid is Lexicographically Shellable
}

\author{
Mahir Bilen $\mathrm{Can}^{1}$ \\ ${ }^{1}$ Tulane University, New Orleans; mahirbilencan@gmail.com
}

June 26, 2019

\begin{abstract}
We prove that the Bruhat-Chevalley-Renner order on the rook monoid is ELshellable. We determine the homeomorphism type of the associated order complex.
\end{abstract}

Keywords: Rook monoid, Bruhat-Chevalley-Renner order, EL-shellability, homeomorphism type.

MSC: 05E45, 20M32

\section{Introduction}

Let $n$ be a positive integer, and let $[n]$ denote the set $\{1, \ldots, n\}$. Let $P$ be a finite graded poset of rank $n$, with minimum and maximum elements denoted by $\hat{0}$ and $\hat{1}$, respectively. We denote by $C(P)$ the set of pairs $(x, y)$ from $P \times P$ such that $y$ covers $x$. The poset $P$ is called lexicographically shellable, or EL-shellable, if there exists a map $f: C(P) \rightarrow[n]$ such that

(1) in every interval $[x, y] \subseteq P$, there exists a unique maximal chain $\mathfrak{c}: x=x_{0}<x_{1}<$ $\cdots<x_{k+1}=y$ such that $f\left(x_{i}, x_{i+1}\right) \leq f\left(x_{i+1}, x_{i+2}\right)$ for $i=0, \ldots, k-1$;

(2) the sequence $f(\mathfrak{c}):=\left(f\left(x, x_{1}\right), \ldots, f\left(x_{k}, y\right)\right)$ of the unique chain $\mathfrak{c}$ of $(1)$ is lexicographically first among all sequences of the form $f(\mathfrak{d})$, where $\mathfrak{d}$ is a maximal chain in $[x, y]$.

Introduced by Björner in [2], the notion of lexicographic shellability has important topological consequences. For example, it is known that if the poset is lexicographically shellable, then the order complex of the poset is a shellable simplicial complex.

In this paper, we are concerned with the question of lexicographic shellability of the Bruhat-Chevalley-Renner order on the monoid of injective partial transformations, which is called the rook monoid, and denoted by $R_{n}$. By definition, $R_{n}$ is the finite monoid consisting of $n \times n$ 0/1 matrices with at most one 1 in each row and in each column. To define the Bruhat-Chevalley-Renner order, we first explain the role of matrices for $R_{n}$. 
Let $M_{n}$ denote the algebraic monoid of all $n \times n$ matrices with entries in $\mathbb{C}$, and let $G L_{n}$ denote its unit-group. We denote by $B_{n}$ the Borel subgroup, which consists of invertible upper triangular elements from $G L_{n}$. We consider the action

$$
\begin{aligned}
\left(B_{n} \times B_{n}\right) \times M_{n} & \longrightarrow M_{n} \\
\left(\left(b_{1}, b_{2}\right), m\right) & \longmapsto b_{1} m b_{2}^{-1}
\end{aligned}
$$

By Bruhat-Chevalley decomposition, the orbits of the restriction of this action to $G L_{n}$ are parametrized by the symmetric group on $n$ letters, denoted by $S_{n}$. Then the BruhatChevalley order on $S_{n}$ is defined by

$$
x \leq y \Longleftrightarrow B_{n} x B_{n} \subseteq \overline{B_{n} y B_{n}} \text {, where } x, y \in S_{n} .
$$

Here, the bar on the orbit indicates the Zariski closure in $G L_{n}$. More generally, the decomposition of $M_{n}$ into $B_{n} \times B_{n}$-orbits is parametrized by the rook monoid,

$$
M_{n}=\bigsqcup_{r \in R_{n}} B_{n} r B_{n},
$$

and the Bruhat-Chevalley-Renner order on $R_{n}$ is defined by

$$
r \leq t \Longleftrightarrow B_{n} r B_{n} \subseteq \overline{B_{n} t B_{n}} \text {, where } r, t \in R_{n},
$$

see [14]. In (1.3), the bar indicates the Zariski closure in $M_{n}$.

We are now ready to state our main results, and explain how we structured our paper. In Section 2, we introduce our notation and review in more detail the Bruhat-Chevalley-Renner order on the rook monoid as well as on the symmetric group. In particular, we review a result of Edelman, which states that the Bruhat-Chevalley order on symmetric groups is lexicographically shellable. In Section 3, by describing an explicit labeling of the edges of the Hasse diagram of the Bruhat-Chevalley-Renner order on $R_{n}$, we state our main result. Its proof will be given in Section 4 .

Theorem 1.4. If $n$ is a positive integer, then $\left(R_{n}, \leq\right)$ is an EL-shellable poset.

The order complex of a poset $P$ is the abstract simplicial complex whose closed sets are the finite chains in $P$. In the final section of our paper, that is Section 5, we consider the order complexes of intervals in $R_{n}$. We finish our paper by proving the second main result of our paper.

Theorem 1.5. The order complex of an open interval in $R_{n}$ is homeomorphic to a sphere or to a ball. In particular, $\Delta\left(R_{n}\right)$ triangulates a ball of dimension $n^{2}$.

Acknowledgements. We thank Lex Renner for teaching us the theory of linear algebraic monoids. We thank the referees whose comments and suggestions greatly improved the quality of our paper. In particular, we are grateful to the referee who showed us another EL-labeling on the rook monoid. Finally, we thank Michelle Wachs and Warut Thawinrak. 


\section{Background}

\subsection{Reductive monoids and Bruhat-Chevalley-Renner order.}

Let $G$ be a connected reductive complex algebraic group. We fix a maximal torus $T$, and a Borel subgroup $B$ in $G$ such that $T \subset B \subset G$. Then the Weyl group of $(G, T)$ is the quotient group $W:=N_{G}(T) / T$, where $N_{G}(T)$ is the normalizer of $T$ in $G$. Note that for the pair $\left(G L_{n}, T\right)$, where $T$ is the maximal torus of diagonal matrices in $G L_{n}$, the Weyl group is isomorphic to the symmetric group, $S_{n}$. The Bruhat-Chevalley order on a Weyl group $W$ is defined by $w \leq v \Longleftrightarrow B w B \subseteq \overline{B v B}$ for $v, w \in W$. It is well-known that such orders are lexicographically shellable, see $[3,8,11]$.

An algebraic monoid is an algebraic variety $M$ together with an associative multiplication morphism $m: M \times M \rightarrow M$ such that there is a neutral element. For an algebraic monoid $M$, the unit-group, that is the group of invertible elements of $M$, has the structure of an algebraic group. A reductive monoid is an algebraic monoid $M$ such that the unit-group of $M$ is a connected reductive algebraic group.

Interesting examples of reductive monoids are built from representations. Let $\rho: G_{0} \rightarrow$ $G L(V)$ be a rational representation of a semisimple algebraic group $G_{0}$. Let us denote by $\mathbb{C}^{*}$ the multiplicative group of nonzero scalar matrices in $\operatorname{End}(V)$. Then the Zariski closure $M:=\overline{\mathbb{C}^{*} \cdot \rho\left(G_{0}\right)}$ in $\operatorname{End}(V)$ has the structure of a reductive monoid. The normality of such monoids is intimately related to the highest weight of the representation, see [6].

Let $G$ denote the unit-group of a reductive monoid $M$, and let $T \subset B$ be a maximal torus and a Borel subgroup in $G$. It is shown in [14] that there is a decomposition of $M$ into double cosets of $B, M=\bigsqcup_{r \in R} B \dot{r} B$, where $R:=\overline{N_{G}(T)} / T$ and $\dot{r} \in \overline{N_{G}(T)}$. The parametrizing object $R$, which is a finite monoid, is called the Renner monoid of $M$. The Bruhat-Chevalley-Renner order on $R$ is defined by

$$
w \leq v \Longleftrightarrow B w B \subseteq \overline{B v B} \text { for } v, w \in R .
$$

If $\rho: G_{0} \rightarrow G L\left(K^{n}\right)$ is the defining representation of $G_{0}=S L_{n}$, then the Renner monoid $R$ is isomorphic to the rook monoid $R_{n}$. The Weyl group $W$ of $(G, T)$ is the unit-group of $R$, and the Bruhat-Chevalley order on $W$ extends to the Bruhat-Chevalley-Renner order on $R$.

There is a cross section lattice of idempotents, denoted by $\Lambda$, in $R$ such that

$$
M=\bigsqcup_{e \in \Lambda} G e G \text { and } R=\bigsqcup_{e \in \Lambda} W e W .
$$

It is shown by Putcha in [13] that the diagonal subposet $W e W \subseteq R(e \in \Lambda)$ is a retract of a shellable poset. Note that, if $\Lambda \backslash\{0\}$ has a unique minimal element, for example when $M$ is the Zariski closure of an irreducible representation of a connected reductive group, then the cross section lattice is an (upper) semimodular lattice. In this case, $\Lambda$ is shellable. We expect that the Renner monoid of a normal reductive monoid is EL-shellable.

For more information about linear algebraic monoids, we recommend the resources $[12$, 15, 16]. 


\subsection{Lexicographic shellability}

Let $P$ be a graded poset of rank $n$ with a maximum and a minimum element, denoted by $\hat{1}$ and $\hat{0}$ respectively. Then all maximal chains of $P$ have equal length $n$. If $x$ and $y$ are two elements from $P$ such that $y$ covers $x$, then we will write $x \lessdot y$. We denote by $C(P)$ the set of covering relations in $P, C(P)=\{(x, y) \in P \times P: x \lessdot y\}$. An edge-labeling on $P$ is a map of the form $f=f_{P, \Gamma}: C(P) \rightarrow \Gamma$, where $\Gamma$ is a totally ordered set. The Jordan-Hölder sequence (with respect to $f$ ) of a maximal chain $\mathfrak{c}: x_{0} \lessdot x_{1} \lessdot \cdots \lessdot x_{n-1} \lessdot x_{n}$ of $P$ is the $n$-tuple $f(\mathfrak{c})=\left(f\left(x_{0}, x_{1}\right), f\left(x_{1}, x_{2}\right), \ldots, f\left(x_{n-1}, x_{n}\right)\right) \in \Gamma^{n}$. We fix an edge labeling, denoted $f$, and we fix a maximal chain $\mathfrak{c}: x_{0}<x_{1}<\cdots<x_{n}$. We call both of the maximal chain $\mathfrak{c}$ and its image $f(\mathfrak{c})$ increasing if the following inequalities hold true:

$$
f\left(x_{0}, x_{1}\right) \leq f\left(x_{1}, x_{2}\right) \leq \cdots \leq f\left(x_{n-1}, x_{n}\right) .
$$

Let $k$ be a positive integer. We consider the lexicographic order on the $k$-fold cartesian product, $\Gamma^{k}=\Gamma \times \cdots \times \Gamma$. An edge labeling $f: C(P) \rightarrow \Gamma$ is called an EL-labeling if

1. in every interval $[x, y] \subseteq P$ of rank $k>0$ there exists a unique maximal chain $\mathfrak{c}$ such that $f(\mathfrak{c}) \in \Gamma^{k}$ is increasing,

2. the Jordan-Hölder sequence $f(\mathfrak{c}) \in \Gamma^{k}$ of the unique chain $\mathfrak{c}$ from (1) is the smallest among the Jordan-Hölder sequences of maximal chains $x=x_{0} \lessdot x_{1} \lessdot \cdots \lessdot x_{k}=y$.

A poset $P$ is called EL-shellable if it has an EL-labeling.

Remark 2.1. There are various lexicographic shellability conditions in the literature, and the EL-shellability that is defined here is among the stronger ones, see [19, Section 3.2]. A deep relationship between EL-shellability of a Coxeter group $W$ and the Kazhdan-Lusztig theory of the Hecke algebra associated with $W$ is found by Dyer in [7].

\subsection{The symmetric group}

We already introduced the notation $S_{n}$ for the set of all permutations of $[n]$. We represent the elements of $S_{n}$ in one-line notation, $w=\left(w_{1}, \ldots, w_{n}\right) \in S_{n}$, so that $w(i)=w_{i}$. It is well-known that $S_{n}$ is a graded poset with respect to Bruhat-Chevalley order. Let $B_{n}$ denote, as before, the subgroup of invertible upper triangular matrices in $G L_{n}$. The grading on $S_{n}$ is given by the length function

$$
\ell(w)=\operatorname{dim}\left(B_{n} w B_{n}\right)-\operatorname{dim}\left(B_{n}\right)=i n v(w),
$$

where

$$
\operatorname{inv}(w)=\left|\left\{(i, j): 1 \leq i<j \leq n, w_{i}>w_{j}\right\}\right| .
$$

Note that $\operatorname{dim} B_{n}=\left(\begin{array}{c}n+1 \\ 2\end{array}\right)$.

The Bruhat-Chevalley order on $S_{n}$ is the partial order generated by the transitive closures of the following covering relations: $x=\left(a_{1}, \ldots, a_{n}\right)$ is covered by $y=\left(b_{1}, \ldots, b_{n}\right)$ if $\ell(y)=$ $\ell(x)+1$, and 
1. $a_{k}=b_{k}$ for $k \in\{1, \ldots, \widehat{i}, \ldots, \widehat{j}, \ldots, n\}$ (hat means omit those numbers),

2. $a_{i}=b_{j}, a_{j}=b_{i}$, and $a_{i}<a_{j}$.

Let $\Gamma=[n] \times[n]$ denote the poset of pairs, ordered lexicographically: $(i, j) \leq(r, s)$ if $i<r$, or $i=r$ and $j<s$. For permutations $x, y \in S_{n}$ given as above, define $f(x, y):=\left(a_{i}, a_{j}\right)$. In Figure 2.1, we depict the corresponding EL-labeling for $S_{3}$.

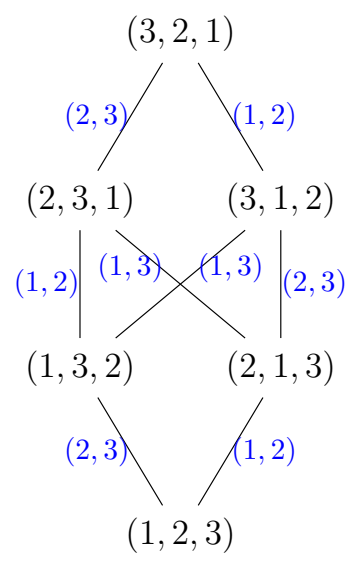

Figure 2.1: EL-labeling of $S_{3}$

Theorem 2.4. ([8]) Let $f$ denote the edge-labeling on the Bruhat-Chevalley order on $S_{n}$ as defined in the previous paragraph. Then $S_{n}$ is an EL-shellable poset with respect to $f$.

\subsection{The rook monoid}

The rook monoid $R_{n}$ is a graded poset (see [14]) and its rank function is given by

$$
\ell(x)=\operatorname{dim}\left(B_{n} x B_{n}\right), x \in R_{n} .
$$

There is a combinatorial formula for $\ell(x), x \in R_{n}$ that is similar to (2.2). To explain, we represent the elements of $R_{n}$ by $n$-tuples. Let $x=\left(x_{i j}\right) \in R_{n}$, and we define the sequence $\left(a_{1}, \ldots, a_{n}\right)$ by

$$
a_{j}= \begin{cases}0 & \text { if the } j \text {-th column consists of zeros } \\ i & \text { if } x_{i j}=1\end{cases}
$$

By abuse of notation, we denote both of the matrix and the corresponding sequence $\left(a_{1}, \ldots, a_{n}\right)$ by $x$. For example, the associated sequence of the partial permutation matrix

$$
x=\left(\begin{array}{llll}
0 & 0 & 0 & 0 \\
0 & 0 & 0 & 0 \\
1 & 0 & 0 & 0 \\
0 & 0 & 1 & 0
\end{array}\right)
$$


is $x=(3,0,4,0)$.

Let $x=\left(a_{1}, \ldots, a_{n}\right)$ be an element from $R_{n}$. A pair of indices $(i, j)$ with $1 \leq i<j \leq n$ is called a coinversion pair for $x$ if $0<a_{i}<a_{j}$. We denote the number of coinversion pairs of $x$ by coinv $(x)$.

Example 2.6. If $x$ is given by $x=(4,0,2,3)$, then the only coinversion pair for $x$ is $(3,4)$.

In [4], it is shown that the dimension $\ell(x)=\operatorname{dim}\left(B_{n} x B_{n}\right)$ of an orbit $B_{n} x B_{n}, x \in R_{n}$ is given by

$$
\ell(x)=\left(\sum_{i=1}^{n} a_{i}^{*}\right)-\operatorname{coinv}(x), \text { where } a_{i}^{*}= \begin{cases}a_{i}+n-i, & \text { if } a_{i} \neq 0 \\ 0, & \text { if } a_{i}=0\end{cases}
$$

We reformulate (2.7) as follows:

Proposition 2.8. Let $x=\left(a_{1}, \ldots, a_{n}\right) \in R_{n}$. Then we have $\ell(x)=\sum a_{i}+i n v(x)$, where $\operatorname{inv}(x)=\left|\left\{(i, j): 1 \leq i<j \leq n, a_{i}>a_{j}\right\}\right|$.

Proof. Since $\operatorname{coinv}(x)=\sum_{i=1}^{n} c_{i}$, where $c_{i}$ is the number of $j \in[n]$ such that $i<j$ and $0<a_{i}<a_{j}$, we can rewrite (2.7) as follows:

$$
\ell(x)=\sum_{i=1}^{n}\left(a_{i}^{*}-c_{i}\right)=\sum_{i=1}^{n}\left(a_{i}+d_{i}^{*}\right)=\sum_{i=1}^{n} a_{i}+\sum_{i=1}^{n} d_{i}^{*},
$$

where

$$
d_{i}^{*}= \begin{cases}n-i-c_{i}, & \text { if } a_{i} \neq 0 \\ 0, & \text { if } a_{i}=0\end{cases}
$$

Observe now that $d_{i}^{*}$ is equal to the number of $j>i$ such that $a_{i}>a_{j} \geq 0$. This finishes the proof.

The following consequence of Proposition 2.8 agrees with equation (2.2).

Corollary 2.9. Let $w=\left(a_{1}, \ldots, a_{n}\right) \in S_{n}$ be a permutation. Then the length of $w$ as an element of the poset $\left(R_{n}, \leq\right)$ is given by $\ell(w)=\left(\begin{array}{c}n+1 \\ 2\end{array}\right)+i n v(w)$.

The first concrete description of the Bruhat-Chevalley-Renner order on $R_{n}$ is given in [10, Theorem 3.8]. We state it here for convenience.

Theorem 2.10. Let $x=\left(a_{1}, \ldots, a_{n}\right), y=\left(b_{1}, \ldots, b_{n}\right) \in R_{n}$. The Bruhat-Chevalley order on $R_{n}$ is the smallest partial order on $R_{n}$ generated by declaring $x \leq y$ if either

1. there exists an $1 \leq i \leq n$ such that $b_{i}>a_{i}$ and $b_{j}=a_{j}$ for all $j \neq i$, or

2. there exist $1 \leq i<j \leq n$ such that $b_{i}=a_{j}, b_{j}=a_{i}$ with $b_{i}>b_{j}$, and for all $k \notin\{i, j\}$, $b_{k}=a_{k}$. 
The covering relations of the order are analyzed in detail in [4], and the following two lemmas are useful in this analysis. The original statement of the first lemma in [4] had a minor gap, which was pointed out to us by Michelle Wachs. We thank Warut Thawinrak, who helped us to state the lemma in a better way. For completeness, we present the corrected version of the lemma and its proof here.

Lemma 2.11. Let $x=\left(a_{1}, \ldots, a_{n}\right)$ and $y=\left(b_{1}, \ldots, b_{n}\right)$ be elements of $R_{n}$. We assume that $a_{k}=b_{k}$ for all $k=\{1, \ldots, \hat{i}, \ldots, n\}$ and that $a_{i}<b_{i}$. Then, $\ell(y)=\ell(x)+1$ if and only if either

1. $0=a_{i}$, and there exists a sequence of indices $1 \leq j_{1}<\cdots<j_{s}<i$ such that the set $\left\{a_{j_{1}}, \ldots, a_{j_{s}}\right\}$ is equal to $\{1, \ldots, s\}, b_{i}=s+1$, and $b_{j}=a_{j}>0$ for $j>i$, or

2. $0<a_{i}$, and there exists a sequence of indices $1 \leq j_{1}<\cdots<j_{s}<i$ such that the set $\left\{a_{j_{1}}, \ldots, a_{j_{s}}\right\}$ is equal to $\left\{a_{i}+1, \ldots, a_{i}+s\right\}$, and $b_{i}=a_{i}+s+1$.

Proof. Let $x, y \in R_{n}$ be two rooks as in the hypothesis, and we assume that $\ell(y)=\ell(x)+1$. By Theorem 2.10, we know that $x \lessdot y$. Obviously, either $b_{i}=a_{i}+1$, or $b_{i}>a_{i}+1$. In the former case, we have two possibilities: $a_{i}=0$, or $a_{i}>0$. In the former case, it follows from Proposition 2.8 that, unless $a_{j}=b_{j}>0$ for all $j>i$, the equality $\ell(y)=\ell(x)+1$ is not true. In the latter case, there is nothing to say. In conclusion, if $b_{i}=a_{i}+1$, then 1 . holds true. On the other hand, if it is true that $b_{i}=a_{i}+d$ for some $d>1$, then more analysis is needed;

$$
\begin{aligned}
\ell(y) & =\sum_{j=1}^{n} b_{j}^{*}-\operatorname{coinv}(y) \\
& =\left(\sum_{j=1, j \neq i}^{n} a_{j}^{*}\right)+b_{i}^{*}-\operatorname{coinv}(y) \\
& =\left(\sum_{j=1, j \neq i}^{n} a_{j}^{*}\right)+a_{i}+d+n-i-\operatorname{coinv}(y) \\
& =\left(\sum_{j=1}^{n} a_{j}^{*}\right)+d-\operatorname{coinv}(y) \\
& =\ell(x)+d+\operatorname{coinv}(x)-\operatorname{coinv}(y) .
\end{aligned}
$$

Hence, $d+\operatorname{coinv}(x)-\operatorname{coinv}(y)=1$, or coinv $(y)-\operatorname{coinv}(x)=d-1$. We inspect the difference $\operatorname{coinv}(x)-\operatorname{coinv}(y)$. If $(k, i)$ with $k<i$ is a coinversion for $x$, then it stays to be a coinversion for $y$. Clearly, this is also true for the pairs of the form $(k, l)$ where $k<i<l$, or $i<k<l$, or $k<l<i$. Therefore, the difference between $\operatorname{coinv}(y)$ and coinv $(x)$ occurs at the pairs of the form

1. $(k, i), k<i$ such that $a_{i}<a_{k}<b_{i}$, or

2. $(i, l), i<l$, such that $a_{i}<a_{l}<b_{i}$. 
In the first case, some new coinversions are added, and in the second case some coinversions are deleted. Let us call the number of pairs of the first type by $n_{1}$, and we call the number of pairs of the second type by $n_{2}$. Then, $\operatorname{coinv}(y)=\operatorname{coinv}(x)+n_{1}-n_{2}$, or $\operatorname{coinv}(y)-\operatorname{coinv}(x)=$ $n_{1}-n_{2}$. We know that $0 \leq n_{1}, n_{2} \leq d-1$ since $b_{i}=a_{i}+d$. Hence, we have that $n_{1}=d-1$, and that $n_{2}=0$. Therefore, the following is true: Any $a_{k}$ between $a_{i}$ and $a_{i}+d=b_{i}$ appears before the $i$-th position. This completes the proof of " $\Rightarrow$ " direction of our lemma.

Next, we prove the " $\Leftarrow$ " direction. If 1 . holds true, then $b_{i}=a_{i}+1$. In this case, it is straightforward to check that $\ell(y)=\ell(x)+1$. So, we assume that there exists a sequence of indices, $1 \leq j_{1}<\cdots<j_{s}<i$, such that the set $\left\{a_{j_{1}}, \ldots, a_{j_{s}}\right\}$ is equal to $\left\{a_{i}+1, \ldots, a_{i}+s\right\}$, and $b_{i}=a_{i}+s+1$. Then

$$
\begin{aligned}
\ell(y) & =\sum_{j=1}^{n} b_{j}^{*}-\operatorname{coinv}(y) \\
& =\left(\sum_{j=1, j \neq i}^{n} a_{j}^{*}\right)+b_{i}^{*}-\operatorname{coinv}(y) \\
& =\left(\sum_{j=1, j \neq i}^{n} a_{j}^{*}\right)+a_{i}+s+1+n-i-\operatorname{coinv}(y) \\
& =\left(\sum_{j=1}^{n} a_{j}^{*}\right)+s+1-\operatorname{coinv}(y) .
\end{aligned}
$$

Now it suffices to show that $\operatorname{coinv}(y)=s+\operatorname{coinv}(x)$. Observe that, when we replace $a_{i}$ by $b_{i}$, the set of pairs, $\left\{\left(j_{k}, i\right) \mid k=1, \ldots, s\right\}$, whose elements are non-coinversion pairs for $x$, become coinversion pairs for $y$. Also, if we replace the entry $a_{i}$ by $b_{i}$, then the coinversion pairs for $x$ of the form $(l, i)$ or $(i, l)$ (where $l \neq j_{k}$ ) are coinversion pairs for $y$ as well. Therefore, we see that $\operatorname{coinv}(y)=s+\operatorname{coinv}(x)$, hence that $\ell(y)=\ell(x)+1$. This finishes the proof of our lemma.

Example 2.12. Let $x=(4,0,5,0,3,1)$ and $y=(4,0,5,0,6,1)$. Then $\ell(x)=21$ and $\ell(y)=22$. If $z=(4,0,5,0,3,2)$, then $\ell(z)=22$.

Lemma 2.13. Let $x=\left(a_{1}, \ldots, a_{n}\right)$ and $y=\left(b_{1}, \ldots, b_{n}\right)$ be two elements of $R_{n}$. Suppose that $a_{j}=b_{i}, a_{i}=b_{j}$ and $b_{j}<b_{i}$ where $i<j$. Furthermore, suppose that for all $k \in$ $\{1, \ldots \widehat{i}, \ldots, \widehat{j}, \ldots, n\}, a_{k}=b_{k}$. Then, $\ell(y)=\ell(x)+1$ if and only if for $s=i+1, \ldots, j-1$, either $a_{j}<a_{s}$, or $a_{s}<a_{i}$.

Example 2.14. Let $x=(2,6,5,0,4,1,7)$ and $y=(4,6,5,0,2,1,7)$. Then $\ell(x)=35$ and $\ell(y)=36$. If $z=(7,6,5,0,4,1,2)$, then $\ell(z)=42$.

\section{An EL-labeling of $R_{n}$}

Let $x$ and $y$ be two elements from $R_{n}$ such that $x \lessdot y$. We call $x \lessdot y$ a type 1 covering relation if it is as in Lemma 2.11, and we call it a type 2 covering relation if it is as in Lemma 2.13. 
Let $\Gamma$ denote the finite set $\Gamma:=\{0,1, \ldots, n\} \times\{1, \ldots, n\}$, totally ordered with respect to lexicographic ordering. We define $F: C\left(R_{n}\right) \longrightarrow \Gamma$ by

$$
F(x, y)= \begin{cases}\left(a_{i}, b_{i}\right), & \text { if } y \text { covers } x \text { by type } 1 \\ \left(a_{i}, a_{j}\right), & \text { if } y \text { covers } x \text { by type } 2 .\end{cases}
$$

Theorem 3.2. Let $\Gamma=\{0,1, \ldots, n\} \times\{0,1, \ldots, n\}$ and let $F: C\left(R_{n}\right) \longrightarrow \Gamma$ be the edgelabeling as defined as in (3.1). Then $F$ is an EL-labeling for $R_{n}$.

We will prove this theorem in the next section. The complete edge-labeling of $R_{3}$ is shown in Figure 3.1.

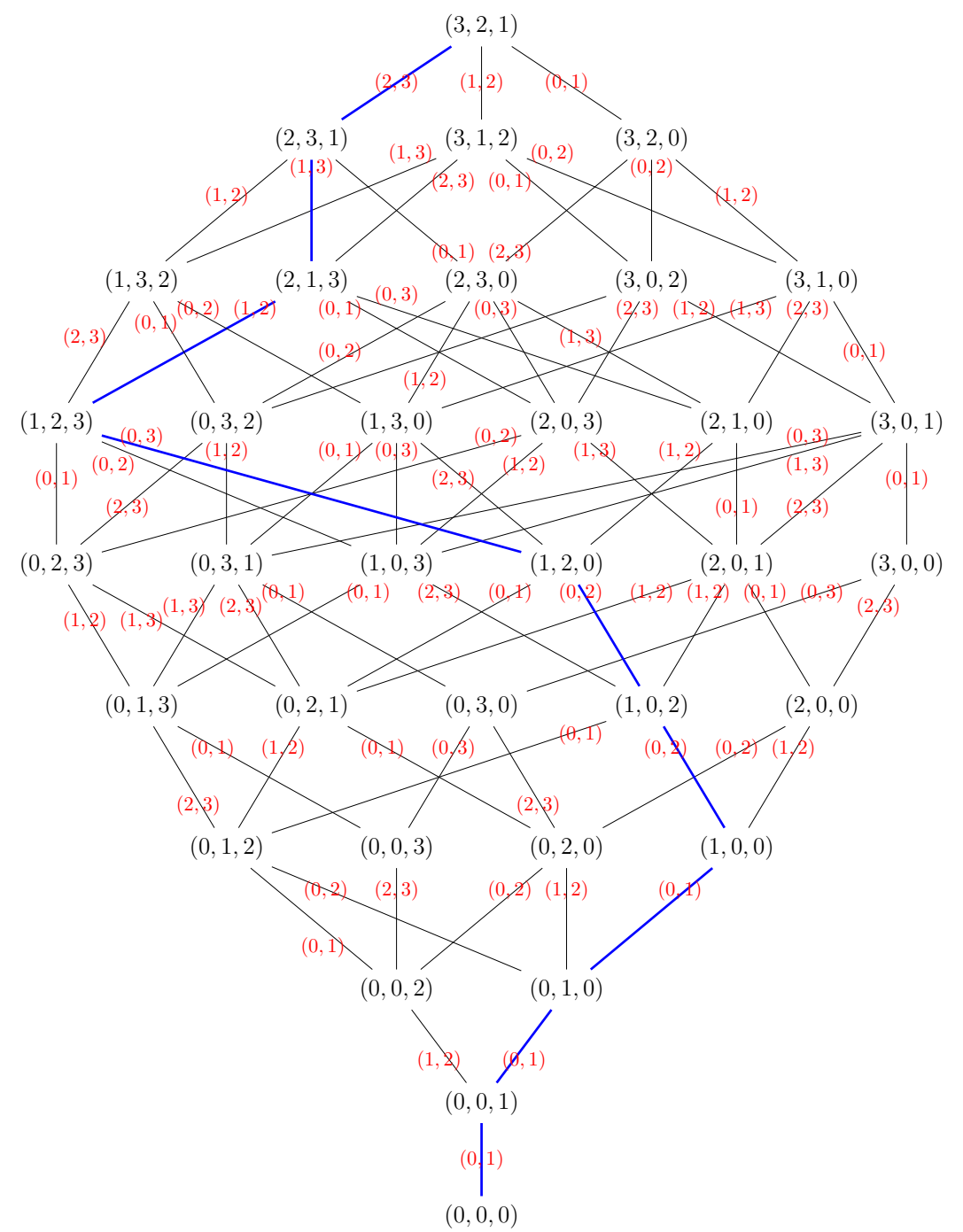

Figure 3.1: EL-labeling of the rook monoid $R_{3}$. 


\section{Proofs}

Let $[x, y]$ be an interval in $R_{n}$, and let $\mathfrak{c}: x=x_{0} \lessdot \cdots \lessdot x_{k}=y$ be a maximal chain in $[x, y]$. The sequence $F(\mathfrak{c}):=\left(F\left(x_{0}, x_{1}\right), \ldots, F\left(x_{k-1}, x_{k}\right)\right)$ will be called the Jordan-Hölder sequence of $\mathfrak{c}$. Here, $F$ is as defined in (3.1).

Example 4.1. Let $x=(0,1,0)$ and $y=(3,1,2)$ be two elements from $R_{3}$. It is easy to check from Figure 3.1 that the maximal chain

$$
\mathfrak{c}: x \lessdot(1,0,0) \lessdot(1,0,2) \lessdot(1,2,0) \lessdot(1,2,3) \lessdot(2,1,3) \lessdot y
$$

is the lexicographically smallest Jordan-Hölder sequence in $[x, y]$. Note that

$$
F(\mathfrak{c})=((0,1),(0,2),(0,2),(0,3),(1,2),(2,3))
$$

is a non-decreasing sequence.

We will describe the lexicographically first chain, denoted by $\mathfrak{c}: x=x_{0} \lessdot \cdots \lessdot x_{k}=y$, in $[x, y]$. Clearly, if $F\left(x, x_{1}\right)=(a, b)$, then $a=\min \{c:(c, d)=F(x, z)$ for $z \in[x, y]$ with $x \lessdot z\}$. First, we assume that the least element in $x$ such that $a_{i} \neq b_{i}$ is nonzero.

Lemma 4.2. If $a_{i}$ is the least element in $x$ such that $a_{i} \notin\left\{0, b_{i}\right\}$, then $x_{1}$ is obtained from $x$ by one of the following covering moves:

- a type 2 covering relation where $a_{i}$ is interchanged with $a_{i}+s+1$ if $a_{i}+s+1$ is after $a_{i}$ but $a_{i}+1, \cdots, a_{i}+s$ appear in $x$ before $a_{i}$;

- a type 1 covering relation that is performed at $a_{i}$.

Proof. We start with a claim. Let $x=\left(a_{1}, a_{2}, \ldots, a_{n}\right)$ and $y=\left(b_{1}, b_{2}, \ldots, b_{n}\right)$ be the one-line representations for $x$ and $y$. If $a_{i}$ denotes the least element such that $a_{i} \notin\left\{0, b_{i}\right\}$, then $a=a_{i}$.

First of all, it is easy to check by using the main result of [4] (that is the Deodhardescription of the Bruhat-Chevalley-Renner ordering on $R_{n}$ ) that $a_{i}<b_{i}$. Secondly, if $a_{i}>a$, then $a_{i} \neq 0$, and therefore, $x$ and $y$ differ from each other at their nonzero entries only. But this immediately yields a contradiction with the minimality of $a_{i}$.

We now assume towards a contradiction that $a_{i}<a$. If the number $b_{i}$ appears in $x$ as an entry $a_{j}$, then we have $i<j$. In this case, we have $a_{j}=b_{i}<b_{j}$ as well. We proceed with this assumption. Then the interchange of $a_{i}$ and $a_{j}$ gives us an element $z$ in $[x, y]$. But this means that there is another element $z^{\prime}$ such that $x \lessdot z^{\prime} \leq z$. Since $z$ agrees with $x$ on its first $i-1$ entries, so does $z^{\prime}$. Furthermore, $z^{\prime}$ can be chosen so that it is obtained from $x$ by moving its $i$-th entry. Then $F\left(x, z^{\prime}\right)$ is of the form $\left(a_{i}, b^{\prime}\right)$ for some $b^{\prime}$. This contradicts the minimality of $(a, b)$.

Let us now assume that $b_{i}$ does not appear in $x$. In this case, we define $z^{\prime}:=\left(c_{1}, \ldots, c_{n}\right)$ by setting $c_{j}$ as $a_{j}$ for $j \neq i$ and by setting $c_{i}$ as the smallest number that is less than or equal to $b_{i}$ and not in $x$. In this case, $z^{\prime}$ covers $x$ with $F\left(x, z^{\prime}\right)=\left(a_{i}, c_{i}\right)$. Since $\left(a_{i}, c_{i}\right)<(a, b)$, we found another contradiction. This finishes the proof of our claim. Notice that the constructions of $z^{\prime}$ in the two cases show also that the covering $x \lessdot x_{1}$ with lexicographically first label is obtained from $x$ as we stated. This finishes the proof of our lemma. 
We now consider the situation where the least element in $x$ such that $a_{i} \neq b_{i}$ is 0 . We have some related notation:

$$
P(x, y):=\left\{i: a_{i}=0,0 \notin\left\{a_{i+1}, b_{i}\right\}\right\} \text { and } Q(x, y):=\left\{a_{i+1}: a_{i}=0,0 \notin\left\{a_{i+1}, b_{i}\right\}\right\} .
$$

We set $q:=\min Q(x, y)$ and $s:=\min \left(\{1, \ldots, n\} \backslash\left\{a_{1}, \ldots, a_{n}\right\}\right)$. Finally, let $a_{r+1}$ be the element in $Q(x, y)$ such that $q=a_{r+1}$.

Lemma 4.3. If $q \leq s$, then $x_{1}$ is obtained from $x$ by interchanging $a_{r}$ with $a_{r+1}$. If $q>s$, then we have the following subcases.

1. If the number of zeros in $x$ and $y$ are the same, then $x_{1}$ is obtained from $x$ by interchanging $a_{r}$ with $a_{r+1}$.

2. If the number of zeros in $x$ and $y$ are different, then we replace $a_{p}$ with $s$, where $p=\max P(x, y)$.

Proof. The fact that these constructions give an element $x_{1}$ such that $x \lessdot x_{1} \leq y$ is easily checked from Lemma 2.11. The minimality of the resulting label, that is $F\left(x, x_{1}\right)$, follows from the construction of $x_{1}$.

Lemmas 4.2 and 4.3 show that the lexicographically first chain in $[x, y]$ is the one that climbs up in the Bruhat-Chevalley-Renner order by moving the smallest "out of place" element in $x_{i}$ (for $i \in\{1, \ldots, k\}$ ) to the left or by suitably incrementing it. It is also clear from the construction of $x_{1}$, hence of $x_{2}, x_{3}$, and so on, that for each $i$ in $\{1, \ldots, k-1\}$ there is only one covering relation whose label is the lexicographically smallest and such that the corresponding label is greater than or equal to the label that is constructed for the previous covering relation. In other words, there is a unique chain $\mathfrak{c}: x \lessdot x_{1} \lessdot x_{2} \lessdot \cdots \lessdot x_{k}=y$ such that $F\left(x, x_{1}\right) \leq F\left(x_{1}, x_{2}\right) \leq F\left(x_{2}, x_{3}\right) \leq \cdots \leq F\left(x_{k-1}, y\right)$. We summarize this observation as follows.

Proposition 4.4. Let $[x, y]$ be an interval in $R_{n}$. Then there is a unique maximal chain $\mathfrak{c}: x=x_{0}<\cdots<x_{k}=y$ in $[x, y]$ whose Jordan-Hölder sequence $F(\mathfrak{c})$ is the lexicographically first sequence. Furthermore, this chain is increasing in the sense that

$$
F\left(x_{0}, x_{1}\right) \leq F\left(x_{1}, x_{2}\right) \leq \cdots \leq F\left(x_{k-1}, x_{k}\right) .
$$

Proof of Theorem 3.2. Let $F: C\left(R_{n}\right) \longrightarrow \Gamma$ denote the edge-labeling that is defined in (3.1). By Proposition 4.4, we know that there is a unique lexicographically first maximal chain in $R_{n}$. In other words, $F$ is an EL-labeling, and hence, $R_{n}$ is an EL-shellable poset.

It is well-known that an interval of length two in a Weyl group consists of four elements. However, for the Renner monoids this is not true in general. In $R_{n}$, already for $n=2$, there are intervals of length two with three elements. We finish this section by proving that in $R_{n}$ an interval of length two has at most four elements. 
Proposition 4.5. Let $[x, y] \subseteq R_{n}$ be an interval of length two. Then, $[x, y]$ is either a chain, or a diamond. In other words, either $[x, y]=\left\{x, x_{1}, x_{1}^{\prime}, y\right\}$ with $x<x_{1} \neq x_{1}^{\prime}<y$, or $[x, y]=\left\{x, x_{1}, y\right\}$ with $x<x_{1}<y$.

Proof. Let $\left(a_{1}, \ldots, a_{n}\right)$ and $\left(b_{1}, \ldots, b_{n}\right)$ denote $x$ and $y$, respectively. Let $J$ denote the set $\left\{i \in[n]: a_{i} \neq b_{i}\right\}$. If $\ell(y)-\ell(x)=2$, then by Lemmas 2.11 and 2.13, we see that $|J| \leq 4$. Therefore, if $z=\left(c_{1}, \ldots, c_{n}\right) \in[x, y]$ is strictly between $x$ and $y$, then the set $\left\{i \in[n]: c_{i} \neq a_{i}\right.$ or $\left.c_{i} \neq b_{i}\right\}$ has at most four elements as well. Arguing case by case, as in Lemmas 4.2 and 4.3 , we see that there are at most two different possibilities for $z$.

\section{$5 \quad$ Final remarks}

Let $P$ be a poset, and let $\widehat{P}$ denote the poset that is obtained from $P$ by adjoining to it $\hat{0}$ (the smallest element) and $\hat{1}$ (the maximal element). We denote by $I(P)$ the set of all intervals in $\widehat{P}$. The Möbius function $\mu: I(P) \longrightarrow \mathbb{Z}$ is an integer valued function, (uniquely) determined by the following conditions:

$$
\mu([x, y])= \begin{cases}1 & \text { if } x=y ; \\ -\sum_{x \leq z<y} \mu([x, z]) & \text { if } x<y .\end{cases}
$$

Let $R_{n, k} \subset R_{n}(0 \leq k \leq n)$ denote the subposet consisting of elements whose rank is $k$. In [1], it is shown that the Möbius function on $I\left(R_{n, k}\right)$ takes values in $\{-1,0,1\}$. When $k=n$, $R_{n, k}$ is the symmetric group, and the Möbius function on $S_{n}$ is well-known (see $[18,17,9]$ ).

The order complex of $P$, denoted by $\Delta(P)$, is the abstract simplicial complex whose faces are the chains in $P$. There is a remarkable topological interpretation of the Möbius function of $\widehat{P}$ in relation with $\Delta(P)$. For $x, y \in P$, we have $\mu((x, y))=\widetilde{\chi}\left(\mathrm{lk}_{x, y}\right)$, where $\mathrm{lk}_{x, y}$ is the order complex of the open interval $(x, y)=\{z \in P: x<z<y\}$, and $\widetilde{\chi}$ is the reduced Euler characteristic of the topological space $\mathrm{lk}_{x, y}$. In particular, $\mu(\widehat{P}):=\mu([\hat{0}, \hat{1}])$ is the reduced Euler characteristic of $\Delta(P)$. In our next result we will determine the homeomorphism type of $\Delta\left(R_{n}\right)$. To this end, let us recall the following important result of Danaraj and Klee.

Lemma 5.1 ([5], page 444). Let $\Delta$ denote a pure shellable simplicial complex in which every codimension one face is contained in at most two facets. If every codimension one face is contained in exactly two facets, then $\Delta$ is homeomorphic to a sphere. Otherwise, $\Delta$ is homeomorphic to a ball.

Theorem 5.2. The order complex $\Delta((x, y))$ of every open interval $(x, y) \subset R_{n}$ is homeomorphic to a sphere, or a ball. In particular, $\Delta\left(R_{n}\right)$ triangulates a ball of dimension $n^{2}$.

Proof. It follows from Proposition 4.5 that every codimension one face of an open interval $(x, y)$ in $R_{n}$ is contained in at most two facets. Thus, by Theorem 3.2 and Lemma 5.1 we see that $\Delta((x, y))$ has the homotopy type of a sphere or a ball.

For our second claim, first we observe that the rank of $\hat{1}$ in $R_{n}$ is $n^{2}$ by Corollary 2.9. Hence, $\operatorname{dim} \Delta\left(R_{n}\right)=n^{2}$. To see that $\Delta\left(R_{n}\right)$ is homeomorphic to a ball, it suffices to notice that $\Delta\left(R_{n}\right)$ has a unique atom, so it is a cone. 
Remark 5.3. Recently, a remarkable generalization of Theorem 5.2 is obtained by Warut Thawinrak in his Undergraduate Honors Thesis at the University of Minnessota-Twin Cities. He describes an effective and easy method for deciding whether or not the Möbius function on an interval $[x, y] \subseteq R_{n}$ takes only nonzero values, and in the non-vanishing situation, he shows that the interval $[x, y]$ is isomorphic to an interval in a suitable symmetric group.

\section{References}

[1] Kürşat Aker, Mahir Bilen Can, and Müge Taşkin. $R$-polynomials of finite monoids of Lie type. Internat. J. Algebra Comput., 20(6):793-805, 2010.

[2] Anders Björner. Shellable and Cohen-Macaulay partially ordered sets. Trans. Amer. Math. Soc., 260(1):159-183, 1980.

[3] Anders Björner and Michelle Wachs. Bruhat order of Coxeter groups and shellability. Adv. in Math., 43(1):87-100, 1982.

[4] Mahir Bilen Can and Lex E. Renner. Bruhat-Chevalley order on the rook monoid. Turkish J. Math., 35(2):1-21, 2011.

[5] Gopal Danaraj and Victor Klee. Shellings of spheres and polytopes. Duke Math. J., 41:443-451, 1974.

[6] Corrado De Concini. Normality and non normality of certain semigroups and orbit closures. In Algebraic transformation groups and algebraic varieties, volume 132 of Encyclopaedia Math. Sci., pages 15-35. Springer, Berlin, 2004.

[7] Matthew J. Dyer. Hecke algebras and shellings of Bruhat intervals. Compositio Math., 89(1):91-115, 1993.

[8] Paul H. Edelman. The Bruhat order of the symmetric group is lexicographically shellable. Proc. Amer. Math. Soc., 82(3):355-358, 1981.

[9] Brant C. Jones. An explicit derivation of the Möbius function for Bruhat order. Order, 26(4):319-330, 2009.

[10] Edwin A. Pennell, Mohan S. Putcha, and Lex E. Renner. Analogue of the BruhatChevalley order for reductive monoids. J. Algebra, 196(2):339-368, 1997.

[11] Robert A. Proctor. Classical Bruhat orders and lexicographic shellability. J. Algebra, 77(1):104-126, 1982.

[12] Mohan S. Putcha. Linear algebraic monoids, volume 133 of London Mathematical Society Lecture Note Series. Cambridge University Press, Cambridge, 1988. 
[13] Mohan S. Putcha. Shellability in reductive monoids. Trans. Amer. Math. Soc., 354(1):413-426 (electronic), 2002.

[14] Lex E. Renner. Analogue of the Bruhat decomposition for algebraic monoids. J. Algebra, 101(2):303-338, 1986.

[15] Lex E. Renner. Linear algebraic monoids, volume 134 of Encyclopaedia of Mathematical Sciences. Springer-Verlag, Berlin, 2005. Invariant Theory and Algebraic Transformation Groups, V.

[16] Louis Solomon. An introduction to reductive monoids. In Semigroups, formal languages and groups (York, 1993), volume 466 of NATO Adv. Sci. Inst. Ser. C Math. Phys. Sci., pages 295-352. Kluwer Acad. Publ., Dordrecht, 1995.

[17] John R. Stembridge. A short derivation of the Möbius function for the Bruhat order. J. Algebraic Combin., 25(2):141-148, 2007.

[18] Daya-Nand Verma. Möbius inversion for the Bruhat ordering on a Weyl group. Ann. Sci. École Norm. Sup. (4), 4:393-398, 1971.

[19] Michelle L. Wachs. Poset topology: tools and applications. In Geometric combinatorics, volume 13 of IAS/Park City Math. Ser., pages 497-615. Amer. Math. Soc., Providence, RI, 2007. 